Springer Link

Proceedings of the 27th International Conference on Systems Engineering, ICSEng

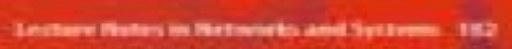

Henry Selwara

Groequerc Chima)

Darbid Iydek Edoses

Proceedings of the

27 th International

Conference

on Systems

Engîneering,

ICSEng 2020

2020

Disininger

International Conference On Systems Engineering

ICSEng 2020: Proceedings of the 27th International Conference on Systems

Engineering, ICSEng 2020 pp 34-48| Cite as

\title{
A Prototype Telepresence Robot for Use in the Investigation of Ebola and Lassa Virus Threatened Villages in Nigeria
}

- Authors

- Authors and affiliations

- S. D. Monk

- E. Jorgensen

- A. McCulloch

- R. Peacock

- I. Sangprachatanaruk

- J. Azeta

- C. A. Bolu

- C. J. Taylor

Conference paper

First Online: 07 January 2021

- 193Downloads

Part of the Lecture Notes in Networks and Systems book series (LNNS, volume 182) 


\section{Abstract}

The article investigates the idea of low-cost, telepresence-based mobile robots for eventual use within villages and rural areas in Nigeria, where diseases such as the Ebola Virus Disease (EVD) and Lassa Haemorrhagic Fever (LHF) are common, yet human intervention is constrained due to the great risk of transmission through bodily fluids. To illustrate the concept and practical issues arising, a systems design approach is taken to identify some of the engineering requirements; and, in the focus of this article, a prototype has been developed at Lancaster University. The robotic device is semihumanoid in that the upper half features two 7-DOF manipulators, designed in part to resemble human operation, while the lower half consists of a four-wheeled base, prioritising ease of operation and reliability over the flexibility offered by a leg-based system.

\section{Keywords}

Telepresence First-Person Viewer Kinect Unsafe environment Ebola

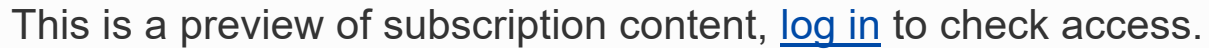

\section{Notes}

Acknowledgements

This work is supported in the UK by the EPSRC project EP/Ro2572X/1.

\section{References}

1. 1.

World Health Organization Ebola Response Roadmap Situation

Reports. http://www.who.int/csr/disease/ebola/situation-reports/archive/en.

Accessed 26 May 2020

2. 2 .

Sanchez, A., Geisbert, T.W., Feldmann H.: Filoviridae: Marburg and Ebola viruses.

Fields Virology. 5th Edn. Lippincott Williams \& Wilkins, Philadelphia, pp. 1409-1448 (2007)Google Scholar

3. 3.

Report of an International Commission: Ebola haemorrhagic fever in Zaire. Bull. World Health Organ. 56(2), 271-293 (1976) Google Scholar

4. 4. 
Tseng, C.P., Chan, Y.J.: Overview of ebola virus disease. J. Chin. Med. Assoc. 78(1), 5155 (2015) CrossRefGoogle Scholar

5. 5 .

Alexander, K.A., Sanderson, C.E., Marathe, M., Lewis, B.L., Rivers, C.M., Shaman, J., Drake, J.M., Lofgren, E., Dato, V.M., Eisenberg, M.C., Eubank, S.: What factors might have led to the emergence of ebola in West Africa?. PLOS Neglected Trop. Dis. 9(6) (2015)Google Scholar

6. 6.

Fauci, A.S.: Ebola-underscoring the global disparities in health care resources. N. Engl. J. Med. 371(12), 1084-1086 (2014)CrossRefGoogle Scholar

7. 7.

Moghadam, S.R.J., Omidi, N., Bayrami, S., Moghadam, S.J., Seyed Alinaghi., S.: Ebola viral disease: a review literature. Asian Pac. J. Trop. Biomed. 5(4), 260-267 (2015)Google Scholar

8. 8.

Ogbu, O., Ajuluchukwu, E., Uneke, C.J.: Lassa fever in West African sub-region: an overview. J. Vector Borne Dis. 44(1), 1 (2007)Google Scholar

9. 9.

World Health Organization, Ebola Virus Disease factsheet. http://www.who.int/newsroom/fact-sheets/detail/ebola-virus-disease. Accessed on 26 May 2020

10.10.

Althaus, C.L., Low, N., Musa, E.O., Shuaib, F., Gsteiger, S.: Ebola virus disease outbreak in Nigeria: transmission dynamics and rapid control. Epidemics 11, 80-84 (2015) CrossRefGoogle Scholar

Weyer, J., Grobbelaar, A., Blumberg, L.: Ebola virus disease: history, epidemiology and outbreaks. Curr. Infect. Dis. Rep. 17(5), 21 (2015)rossRefGoogle Scholar

12. 12.

Sheridan, T.: Human supervisory control of robot systems. In: Proceedings of the IEEE International Conference on Robotics and Automation, vol. 3, pp. 808-812. IEEE (1986) Google Scholar 
Harless, M., Donath, M.: An intelligent safety system for unstructured human/robot interaction. In: Proceedings of the Robots Conference and Exposition, 117-120 (1985)Google Scholar

14. 14

Murphy, R.R.: Rescue robotics for homeland security. Commun. ACM 47(3), 66-68 (2004)CrossRefGoogle Scholar

15.15

Murphy, R.R.: A decade of rescue robots. In: IEEE/RSJ International Conference on Intelligent Robots and Systems, pp. 5448-5449. IEEE (2012)Google Scholar

16. 16

Kristoffersson, A., Eklundh, K.S., Loutfi, A.: Measuring the quality of interaction in mobile robotic telepresence: a pilot's perspective. Int. J. Soc. Robot. 5(1), 89-101 (2013) CrossRefGoogle Scholar

17.17.

Paulos, E., Canny, J.: Designing personal tele-embodiment. In: Proceedings of the IEEE International Conference on Robotics and Automation, pp. 3173-3178 (1998)Google Scholar

18. 18.

Paulos, E., Canny, J.: Social tele-embodiment: understanding presence. Auton. Robots 11(1), 87-95 (2001)zbMATHCrossRefGoogle Scholar

19. 19 .

Adalgeirsson, S.O., Breazeal, C.: MeBot: a robotic platform for socially embodied presence. In: Proceedings of the $5^{\text {th }}$ ACM/IEEE International Conference on HumanRobot Interaction (HRI), Osaka, Japan, pp. 15-22 (2010)Google Scholar

20.20

Burgard, W., Trahanias, P., Hähnel, D., Moors, M., Schulz, D., Baltzakis, H., Argyros, A.: Tele-presence in populated exhibitions through web-operated mobile robots. Auton. Robots 15(3), 309-316 (2003)Google Scholar

Chaudary, B., Paajala, I., Keino, E., Pulli, P.: Tele-guidance based navigation system for the visually impaired and blind persons. In: eHealth vol. 360, pp. 9-16. Springer, Cham (2017)Google Scholar 
22. 22.

Double robotics website. https://www.doublerobotics.com/. Accessed on 26 May 2020

23. 23.

IEEE Spectrum

article. https://spectrum.ieee.org/automaton/robotics/humanoids/toyota-gets-back-

into-humanoid-robots-with-new-thr3. Accessed 26 May 2020

24.24 .

Toyota website. https://newsroom.toyota.co.jp/en/detail/19666346. Accessed 26 May 2020

25.25.

Whitney, J.P., Chen, T., Mars, J., Hodgins, J.K.: A hybrid hydrostatic transmission and human-safe haptic telepresence robot. In: IEEE International Conference on Robotics and Automation, Stockholm, Sweden, pp. 690-695 (2016)Google Scholar

26. 26.

Bodner, J., Wykypiel, H., Wetscher, G., Schmid, T.: First experiences with the da Vinci $^{\mathrm{TM}}$ operating robot in thoracic surgery. Eur. J. Cardio-thoracic Surg. 25(5), 844851 (2004) CrossRefGoogle Scholar

Quinetiq Dragon Runner. https://www.qinetiq-na.com/products/unmanned-

systems/dragon-runner. Accessed 26 May 2020

28.28.

Xsens website. https://www.xsens.com/cases/robo-sally-bomb-disposal-robot. Accessed on 26 May 2020

29.29.

Abe, K., Shiomi, M., Pei, Y.C., Zhang, T.Y., Ikeda, N., Nagai, T.: ChiCaRo: tele-presence robot for interacting with babies and toddlers. Adv. Robot. 32(4), 176-190 (2018)CrossRefGoogle Scholar

30.30 .

Ilias, B., Shukor, S.A., Yaacob, S., Adom, A.H., Razali, M.M.: A nurse following robot with high speed kinect sensor. ARPN J. Eng. Appl. Sci. 9(12), 2454-2459 (2014)Google $\underline{\text { Scholar }}$ 
Ardanuy, P., Otto, C., Head, J., Powell, N., Grant, B., Howard, T.: Telepresence enabling human and robotic space exploration and discovery: antarctic lessons learned. In: Space, Long Beach California, USA, p. 6756 (2005) Google Scholar

Kron, A., Schmidt, G., Petzold, B., Zah, M., Hinterseer, P., Steinbach, E.: Disposal of explosive ordnances by use of a bimanual haptic telepresence system. In: IEEE International Conference on Robotics and Automation, New Orleans, LA, USA, vol. 2, pp. 1968-1973 (2004) Google Scholar

Talha, M., Ghalamzan, E.A.M., Takahashi, C., Kuo, J., Ingamells, W., Stolkin, R.: Towards robotic decommissioning of legacy nuclear plant: Results of human-factors experiments with tele-robotic manipulation, and a discussion of challenges and approaches for decommissioning. In: IEEE International Symposium on Safety, Security, and Rescue Robotics, Lausanne, Switzerland, pp. 166-173 (2016)Google $\underline{\text { Scholar }}$

West, C., Monk, S., Montazeri, A., Taylor, C.J.: A vision-based positioning system with inverse dead-zone control for dual-hydraulic manipulators. In: UKACC 12th International Conference on Control, Sheffield, UK, IEEE (2018) Google Scholar

Tsitsimpelis, I., Taylor, C.J., Lennox, B., Joyce, M.J.: A review of ground-based robotic systems for the characterization of nuclear environments. Progress Nuclear Energy 111, 109-124 (2019) CrossRefGoogle Scholar

36. 36.

Colgate, E., Bicchi, A., Peshkin, M.A., Colgate, J.E.: Safety for physical human-robot interaction. In: Springer Handbook of Robotics pp. 1335-1348. Springer (2008)Google $\underline{\text { Scholar }}$

Kulić, D., Croft, E.: Pre-collision safety strategies for human-robot interaction. Auton. Robots 22(2), 149-164 (2007) CrossRefGoogle Scholar 
RIA/ANSI R15.06-1999 American National Standard for Industrial Robots and Robot Systems-Safety Requirements. American National Standards Institute. New YorkGoogle Scholar

Hussein, M.A., Ali, A.S., Elmisery, F., Mostafa, R.: Motion control of robot by using kinect sensor. Res. J. Appl. Sci. Eng. Tech. 8(11), 1384-1388 (2014)CrossRefGoogle $\underline{\text { Scholar }}$

Marturi, N., Rastegarpanah, A., Takahashi, C., Adjigble, M., Stolkin, R., Zurek, S., Kopicki, M., Talha, M., Kuo, J.A., Bekiroglu, Y.: Advanced robotic manipulation for nuclear decommissioning: a pilot study on tele-operation and autonomy. In: Proceedings of the International Conference on Robotics and Automation for Humanitarian Applications, Kerala, India, pp. 1-8 (2016)Google Scholar

\section{Copyright information}

(C) Springer Nature Switzerland AG 2021

Notes in Networks and Systems, vol 182. Springer, Cham. https://doi.org/10.1007/978-3-030-65796-3_4

- First Online07 January 2021

- DOIhttps://doi.org/10.1007/978-3-030-65796-3_4

- Publisher NameSpringer, Cham

- Print ISBN978-3-030-65795-6

- Online ISBN978-3-030-65796-3

- eBook Packages Intelligent Technologies and RoboticsIntelligent Technologies and Robotics (R0)

- Reprints and Permissions

Chapter

EUR 24.95

Price excludes VAT (Nigeria)

- DOI: $10.1007 / 978-3-030-65796-3 \_4$

$r$

eBook

EUR 160.49

Softcover Book 
Springer Nature

(C) 2020 Springer Nature Switzerland AG. Part of Springer Nature.

Not logged in Not affiliated 165.73.223.226 